\title{
Lobar Ventilation in Patients with COPD Assessed with the Full-Scale Airway Network Flow Model and Xenon-enhanced Dual-Energy CT
}

\author{
Minsuok Kim, PhD • Ozkan Doganay, PhD • Hye Jeon Hwang, MD, PhD • Joon Beom Seo, MD, PhD • \\ Fergus V. Gleeson, FRCP, FRCR, FCCP
}

From the School of Mechanical, Electrical and Manufacturing Engineering, LoughboII rough University, Loughborough LE11 3TU, England (M.K.); Healthy Science Institute, Ege University, Izmir, Turkey (O.D.); Department of Radiology and Research Institute of Radiology, Asan Medical Center, University of Ulsan College of Medicine, Seoul, Republic of Korea (H.J.H., J.B.S.); Department of Oncology, University of Oxford, Oxford, England (F.V.G.); and Department of Radiology, The Churchill Hospital, Oxford University Hospitals NHS Trust, Headington, England (F.V.G.). Received June 2, 2020; revision requested July 24; revision received September 8; accepted October 1. Address correspondence to M.K. (e-mail: m.kim@lboro.ac.uk).

Supported by the Cancer Research UK (grant C5255), Engineering and Physical Sciences Research Council (grant A16466), and NIHR Oxford Biomedical Research Centre.

Conflicts of interest are listed at the end of this article.

See also the editorial by Parraga and Eddy in this issue.

Radiology 2020; 00:1-9 • https://doi.org/10.1148/radiol.2020202485 • Content code: CH

Background: The full-scale airway network (FAN) flow model shows excellent agreement with limited functional imaging data but requires further validation prior to clinical use.

Purpose: To validate the ventilation distributions computed with the FAN flow model with xenon ventilation from xenon-enhanced dual-energy (DE) CT in participants with chronic obstructive pulmonary disease (COPD).

\begin{abstract}
Materials and Methods: In this prospective study, the FAN model extracted structural data from xenon-enhanced DE CT images of men with COPD scanned between June 2012 and July 2013 to compute gas ventilation dynamics. The ventilation distributions on the middle cross-section plane, percentage lobar ventilation, and ventilation heterogeneity quantified by the coefficient of variation (CV) were compared between xenon-enhanced DE CT imaging and the FAN model. The relationship between the ventilation parameters with the densitometry and pulmonary function test results was demonstrated. The agreements and correlations between the parameters were measured using the concordance correlation coefficient and the Pearson correlation coefficient.
\end{abstract}

Results: Twenty-two men with COPD (mean age, 67 years \pm 7 [standard deviation]) were evaluated. The percentage lobar ventilation computed with FAN showed a strong positive correlation with xenon-enhanced DE CT data $(r=0.7, P<.001)$. Ninety-five percent of lobar ventilation CV differences lay within $95 \%$ confidence intervals. Correlations of the percentage lobar ventilation were negative for percentage emphysema (xenon-enhanced DE CT: $r=-0.38, P<.001$; FAN: $r=-0.23, P=.02$ ) but were positive for percentage normal tissue volume (xenon-enhanced DE CT: $r=0.78, P<.001$; FAN: $r=0.45, P<.001$ ). Lung CVs of FAN revealed negative correlations with the spirometry results $\left(\mathrm{CV}_{\mathrm{FAN}}\right.$ vs percentage predicted forced expiratory volume in 1 second: $r=$ $-0.75, P<.001 ; \mathrm{CV}_{\mathrm{FAN}}$ vs ratio of forced expiratory volume in 1 second to forced vital capacity: $r=-0.67, P<.001$ ).

Conclusion: The full-scale airway network modeled lobar ventilation in patients with chronic obstructive pulmonary disease correlated with the xenon-enhanced dual-energy CT imaging data.

(c) RSNA, 2020

Online supplemental material is available for this article.

$c$

hronic obstructive pulmonary disease (COPD) is characterized by long-term airway inflammation and parenchymal destruction that interferes with normal airway flow, causing respiratory impairment. It is a major burden on the health care system, with a prevalence of 251 million cases reported globally in 2016, and it caused 3.17 million deaths in 2015 (1). Recent advances in medical imaging technology have improved the structural assessment of COPD. However, challenges remain in early diagnosis, severity stratification, and effective treatments.

Thoracic CT provides excellent spatial resolution, enabling identification of anatomic and structural changes caused by COPD, but it provides limited functional information. Pulmonary function tests (PFTs) are well established and offer some functional information but only at a whole-lung and nonregional level. The more expensive and time-consuming functional imaging modalities, such as hyperpolarized gas MRI and single-photon emission CT (SPECT) can be used to identify regional functional abnormalities in patients with COPD (2-6), but they are less readily available for routine clinical practice.

Xenon-enhanced dual-energy (DE) CT is a promising tool in the investigation of regional lung ventilation abnormalities in patients with COPD $(7,8)$. It uses two (low and high) photon energy spectra to differentiate inhaled xenon from the natural lung contents of air, blood, and soft tissue (9). Thus, it provides high-spatial-resolution images compared with SPECT (10-12). Xenon-enhanced DE CT is less expensive and more readily available for clinical practice than hyperpolarized gas MRI.

We previously reported on a CT image-based computational model, the full-scale airway network (FAN) flow 


\begin{abstract}
Abbreviations
$\mathrm{COPD}=$ chronic obstructive pulmonary disease, $\mathrm{CV}=$ coefficient of variation, $\mathrm{DE}=$ dual energy, FAN = full-scale airway network, FEV1 = forced expiratory volume in 1 second, $\mathrm{FEV} \%$ = percentage predicted $\mathrm{FEV}_{1}, \mathrm{FVC}=$ forced vital capacity, HPX = hyperpolarized xenon 129 , $\mathrm{PFT}=$ pulmonary function test, $\mathrm{VNC}=$ virtual noncontrast, V-SPECT $=$ ventilation single-photon emission CT

\section{Summary}

Lobar ventilation in patients with chronic obstructive pulmonary disease computed with a full-scale airway network flow model correlated with xenon-enhanced dual-energy CT gas ventilation images.

Key Results

- The percentage lobar ventilation in subjects with chronic obstructive pulmonary disease computed with the full-scale airway network (FAN) model had strong positive linear correlation with xenon-enhanced dual-energy (DE) CT $(r=0.7, P<.001)$.

- Ninety-five percent of lobar ventilation coefficient of variation differences between FAN and xenon-enhanced DE CT lay within 95\% CIs.

- Percentage predicted forced expiratory volume for 1 second negatively correlated with the lung ventilation coefficient of variations assessed with xenon-enhanced DE CT $(r=-0.7, P<.001)$ and FAN $(r=-0.75, P<.001)$.
\end{abstract}

model (13). In patients with COPD, it showed a strong correlation compared with hyperpolarized xenon 129 (HPX) MRI and ventilation SPECT (V-SPECT) imaging data (14). The FAN flow model has the potential to be introduced into clinical practice to probe the role of disease at a microstructural level beyond the limits of conventional imaging analysis (15). In the prior report, CT, HPX MRI, and V-SPECT were performed at different times with inevitable differences in lung volumes and at different planes and section thicknesses, which required image coregistration for FAN model comparisons, inevitably introducing inaccuracies. The present study aims to compare FAN modeled ventilation with xenon-enhanced DE CT imaging data acquired in the same imaging plane (with no need for co-registration or allowance for different lung volumes) and to study the relationship of ventilation with lung densitometry and PFTs in patients with COPD.

\section{Materials and Methods}

\section{Participants and PFT}

This prospective study was approved by the institutional review board of the Asan Medical Center (2019-0451). All procedures were conducted according to the Declaration of Helsinki. Written informed consent for xenon inhalation for the study protocol was obtained from all participants. The authors had full control of the data and information, without industry support or funding to perform this study (Fig 1). The PFTs were conducted by using a spirometer, as recommended by the American Thoracic Society using Vmax 22 (SensorMedics, Yorba Linda, Calif) on the same day as xenon-enhanced DE CT scanning. The PFT results were expressed as the percentage of the measured value to the predicted values of the forced expiratory volume in 1 second $\left(\mathrm{FEV}_{1} \%\right)$ and the ratio of $\mathrm{FEV}_{1}$ to forced vital capacity (FVC)

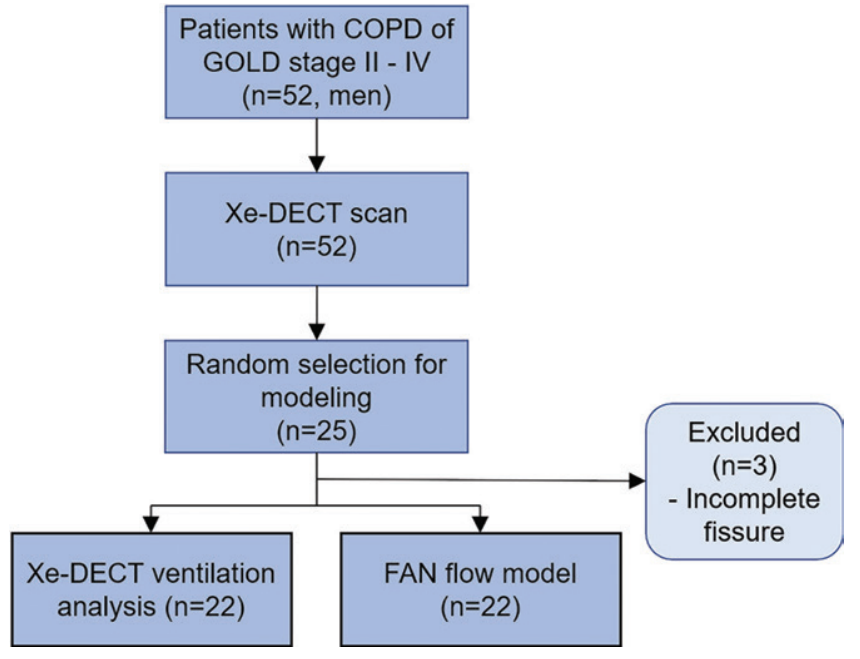

Figure 1: Schematic pipeline of the xenon-enhanced dual-energy CT ( XeDECT) data collection for the full-scale airway network (FAN) flow model analysis. COPD = chronic obstructive pulmonary disease, $G O L D=$ Global Initiative for Chronic Obstructive Lung Disease.

(hereafter, FEV/FVC). Lung volumes, including residual volume, and total lung capacity were measured with body plethysmography (V6200; SensorMedics).

\section{Xenon-enhanced DE CT}

The participants inhaled a mixture of $30 \%$ nonradioactive xenon (30\% xenon, 70\% oxygen) through a gas inhalation system (Zetron V; Anzai Medical, Tokyo, Japan). The concentration of xenon in the expiratory gas and the carbon dioxide $\left(\mathrm{CO}_{2}\right)$ level were monitored with the gas inhalation system throughout the entire study. Xenon-enhanced DE CT scanning with the Somatom Flash CT system (Siemens Healthcare, Forchheim, Germany) was performed in full inspiration 10 seconds after the exhaled xenon concentration reached $25 \%$. The scans covered the full thorax with $512 \times 512$ pixel matrix, $64 \times 0.6 \mathrm{~mm}$ collimation, 89 and $210 \mathrm{~mA}$ (effective) at 140 and $80 \mathrm{kV}$, pitch of 0.55 , and 0.28 -second rotation time. The xenon attenuation maps and virtual noncontrast (VNC) images were constructed from xenon-enhanced DE CT images with a section thickness of $1.5 \mathrm{~mm}$ using kernel D30 and a $1.5-\mathrm{mm}$ increment.

\section{Full-Scale Airway Network Flow Model}

To create the subject-specific model geometries, the surface of pulmonary lobes and large airways (down to generations five to eight) were segmented from the VNC xenon-enhanced DE CT images by using an open-access code (Pulmonary Toolkit; https:// github.com/tomdoel/pulmonarytoolkit). Large-airway centerlines and parenchymal tissue density were extracted from VNC images (16). Small airways beyond the limit of xenon-enhanced DE CT imaging resolution were generated with a branch-growing algorithm in a computational biology open-source library (Chaste; https://github.com/Chaste/Chaste/releases/tag/release_2017.1) from the terminal points of large airways to fill the lumen of lobes (17). Tissue density and lung volume information were applied to determine the acinar boundary conditions. The xenon and $\mathrm{CO}_{2}$ curves of each participant were used to determine the 

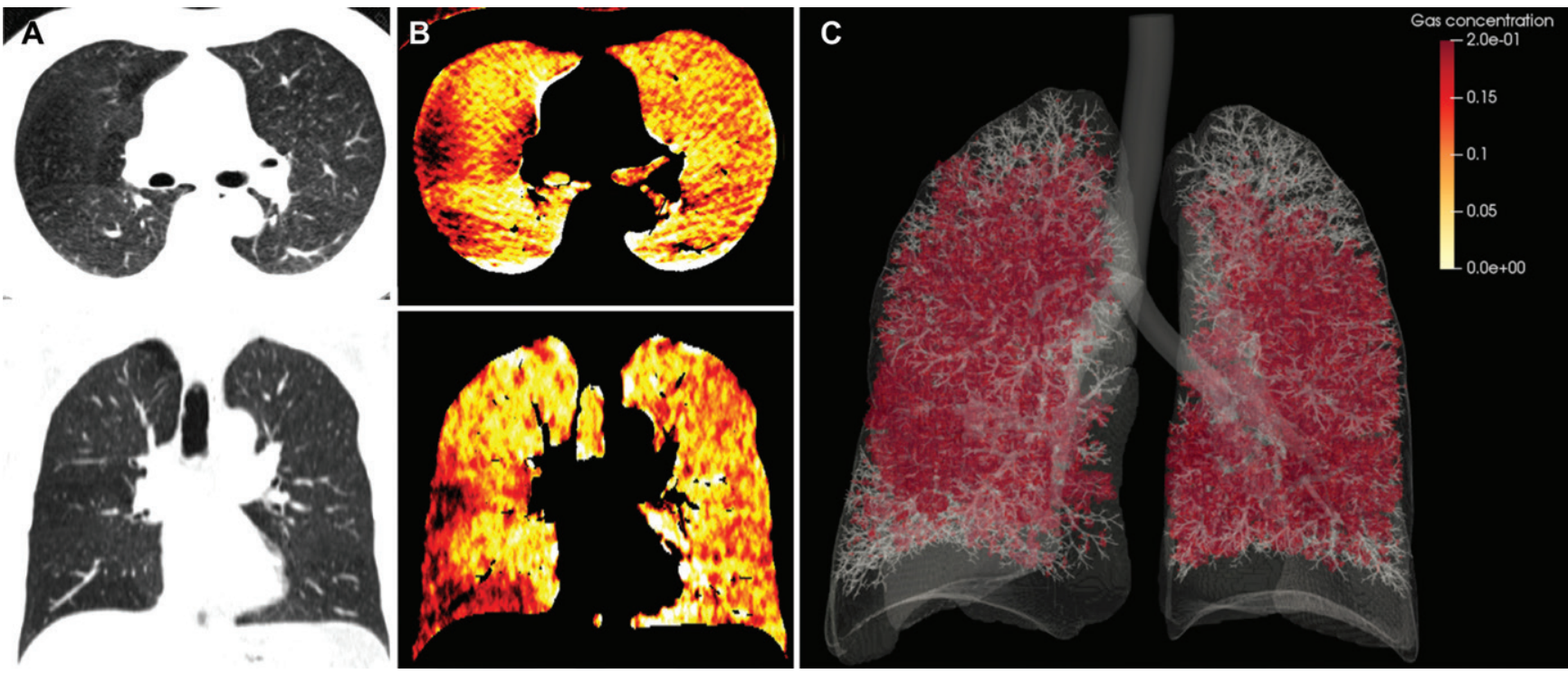

Figure 2: Structural and functional imaging data with illustration of the full-scale airway network (FAN) flow model comparison. A, FAN flow model uses anatomic data in the virtual nonenhanced images to create the airway geometry. B, Xenon-enhanced dual-energy CT images elucidate regional ventilation at full inhalation. C, FANmodeled gas concentration in a lung.

subject-specific conditions of the breathing cycle, the number of breaths while scanning, gas injection, and data acquisition times for FAN modeling. The FAN model computed dynamic airway flow, pressure, and gas concentration. The details of FAN flow modeling were reported previously $(13,14)$. To facilitate lobar analysis, we created tetrahedral volume meshes filling the lobar lumen with an open-source three-dimensional mesh generator (18). Subsequently, gas concentration data from the image and model were mapped onto the volumetric meshes to assess lobar ventilation. Figure 2 shows the images and FAN model geometry.

\section{Data Analysis}

The ventilation distributions on a midtransverse plane and a midcoronal plane passing through the volumetric meshes were analyzed. The ventilation distributions on the middle crosssections of the FAN to those of xenon-enhanced DE CT were independently compared by three authors (M.K., 9 years of modeling experience; O.D., 8 years of image analysis experience; F.G., more than 28 years of clinical radiology experience). We calculated the structural simi-

\begin{tabular}{|c|c|c|c|c|c|c|}
\hline Participant No. & Age (y) & GOLD Stage & $\mathrm{RV}(\%)$ & TLC (\%) & $\mathrm{FEV}_{1 \%}$ & $\mathrm{FEV}_{1} / \mathrm{FVC}$ \\
\hline$\overline{1}$ & 73 & II & 107 & 107 & 56 & 0.41 \\
\hline 2 & 63 & II & 97 & 82 & 52 & 0.58 \\
\hline 3 & 70 & III & 104 & 92 & 49 & 0.42 \\
\hline 4 & 62 & II & 79 & 92 & 55 & 0.46 \\
\hline 5 & 67 & II & 108 & 89 & 51 & 0.47 \\
\hline 6 & 50 & II & 108 & 101 & 54 & 0.47 \\
\hline 7 & 67 & II & 79 & 107 & 68 & 0.44 \\
\hline 8 & 64 & II & 111 & 111 & 57 & 0.42 \\
\hline 9 & 77 & II & 62 & 85 & 75 & 0.55 \\
\hline 10 & 77 & II & 78 & 89 & 75 & 0.57 \\
\hline 11 & 66 & II & 70 & 84 & 63 & 0.54 \\
\hline 12 & 63 & II & 103 & 101 & 57 & 0.48 \\
\hline 13 & 72 & II & 73 & 103 & 68 & 0.44 \\
\hline 14 & 60 & II & 90 & 98 & 67 & 0.56 \\
\hline 15 & 57 & II & 100 & 100 & 50 & 0.43 \\
\hline 16 & 66 & II & 131 & 110 & 57 & 0.49 \\
\hline 17 & 75 & III & 172 & 106 & 42 & 0.50 \\
\hline 18 & 62 & II & 142 & 118 & 68 & 0.54 \\
\hline 19 & 71 & III & 136 & 123 & 30 & 0.21 \\
\hline 20 & 66 & IV & 187 & 107 & 20 & 0.30 \\
\hline 21 & 76 & III & 169 & 121 & 35 & 0.31 \\
\hline 22 & 68 & IV & 175 & 117 & 25 & 0.25 \\
\hline Overall ${ }^{*}$ & $67 \pm 7$ & NA & $112.8 \pm 36.8$ & $102 \pm 12.1$ & $53.4 \pm 15.2$ & $0.45 \pm 0.1$ \\
\hline
\end{tabular}

Note. $-\mathrm{FEV}_{1}=$ forced expiratory volume, $\mathrm{FEV}_{1 \%}=\mathrm{FEV}_{1}$ percentage predicted, $\mathrm{FVC}=$ forced vital capacity, GOLD = Global Initiative for Chronic Obstructive Lung Disease, NA = not applicable, $\mathrm{RV}=$ residual volume, $\mathrm{TLC}=$ total lung capacity.

* Data are mean \pm standard deviation. larity index (Appendix E1 [online]) with Matlab software (MathWorks, Natick, Mass) to quantitatively assess ventilation similarity (19). For regional ventilation, relative percentage lobar ventilation 


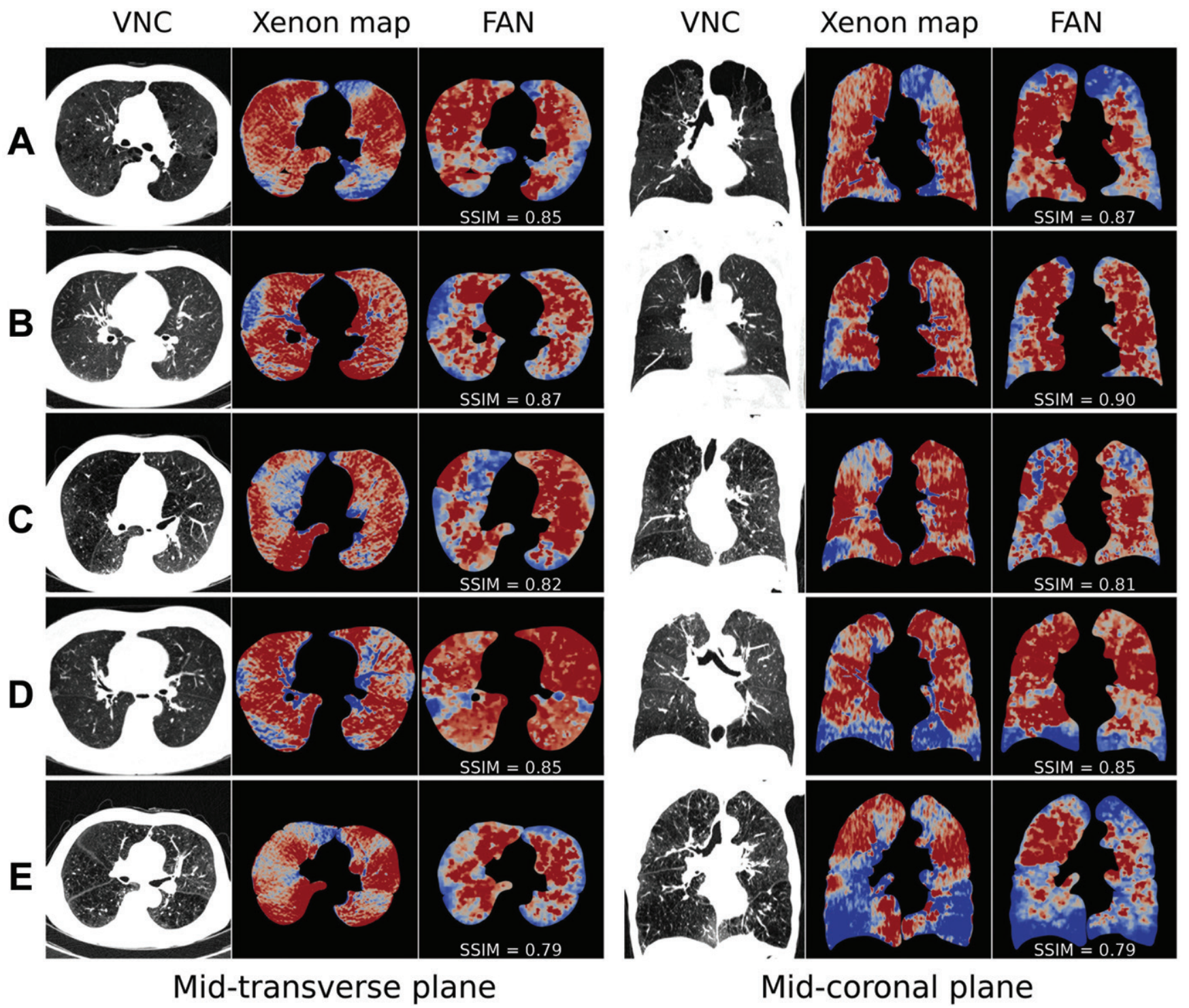

Figure 3: $A-E$, Cross-sectional (midtransverse and midcoronal) images in five participants. Virtual nonenhanced (VNC) images (left) constructed from the 80- and 140-kV xenon-enhanced dual-energy (DE) CT data show the anatomic structure. Xenon map obtained with xenon-enhanced DE CT (middle) and full-scale airway network (FAN) flowmodeled gas concentration (right) shows ventilation distribution. An image quality metric, structural similarity index (SSIM) of the FAN results, was calculated with the xenon map.

based on the xenon maps extracted from xenon-enhanced DE CT imaging data and the FAN-modeled gas density were compared. Additionally, spatial ventilation heterogeneity quantified by using coefficients of variation (CVs) was compared between xenon-enhanced DE CT imaging and the FAN model.

From the densitometry of VNC images, the volume of emphysema in a lobe was identified by using threshold attenuation of less than $-950 \mathrm{HU}(4,20)$, with the remaining lobe volume considered as normal tissue $(21,22)$. Thereafter, lobar analysis was performed to investigate the relationship between the percentage lobar ventilation and structural parameters, such as percentage emphysema ratio and percentage normal tissue ratio. Additionally, the relationship between the emphysema ratio in a whole lung and the CV assessed with xenon-enhanced DE CT and the FAN model were compared. Since PFTs were only available for whole lungs, $\mathrm{FEV} \%$ and $\mathrm{FEV} / \mathrm{FVC}$ ratio were also compared with the whole-lung FAN model and the xenon-enhanced DE CT images.
Statistical Analysis

The concordance correlation was used to measure the percentage ventilation agreement between xenon-enhanced DE $\mathrm{CT}$ and FAN. The linear association of study parameters was quantified using linear regression and Pearson correlation. Bland-Altman analysis also was used to investigate the lobar $\mathrm{CV}$ between xenon-enhanced DE CT and the FAN model. Statistical calculations were conducted with Matlab software (MathWorks, Natick, Mass).

\section{Results}

Participant Demographics

Fifty-two male patients with COPD were recruited from the pulmonology clinic of the Asan Medical Center between February and July 2013 for a previous study of ventilation-perfusion mismatch in patients with COPD (9). We randomly se- 

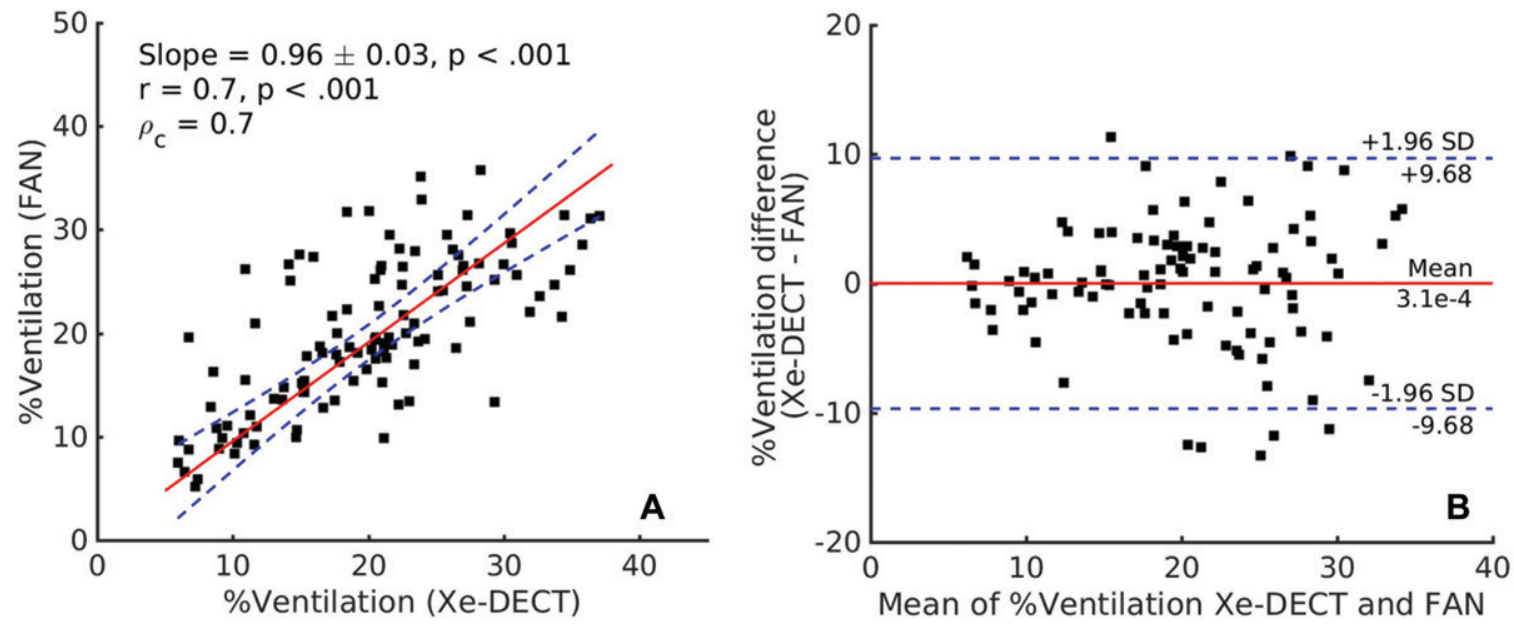

Figure 4: Comparison of percentage lobar ventilation (\%ventilation) between the xenon-enhanced dual-energy CT (Xe-DECT) and full-scale airway network (FAN) flow model. A, Linear regression of relative percentage lobar ventilation assessed with xenon-enhanced dual-energy CT and the FAN model. B, Bland-Altman agreement plot of ventilation heterogeneity quantified by the coefficient of variation

\begin{tabular}{|c|c|c|c|}
\hline Lobe & $\begin{array}{l}\text { Median Ventilation Defect Percentage } \\
\text { Calculated with the FAN Model }\end{array}$ & $\begin{array}{l}\text { Median Ventilation Defect Percentage Calculated } \\
\text { with Ventilation Xenon-enhanced DE CT }\end{array}$ & $P$ Value \\
\hline$\overline{\text { RUL }}$ & $1.87 \pm 1.50$ & $2.05 \pm 1.73$ & .033 \\
\hline RML & $1.49 \pm 0.42$ & $1.76 \pm 0.86$ & .034 \\
\hline RLL & $1.81 \pm 1.08$ & $1.82 \pm 1.44$ & .002 \\
\hline LUL & $2.33 \pm 0.96$ & $2.32 \pm 0.75$ & $<.001$ \\
\hline LLL & $2.44 \pm 0.55$ & $2.89 \pm 0.61$ & $<.001$ \\
\hline
\end{tabular}

Note.-Unless otherwise indicated, data are median $\pm 95 \% \mathrm{CI} . \mathrm{DE}=$ dual energy, FAN $=$ full-scale airway network, LLL $=$ left lower lobe, $\mathrm{LUL}=$ left upper lobe, $\mathrm{RLL}=$ right lower lobe, $\mathrm{RML}=$ right middle lobe, $\mathrm{RUL}=$ right upper lobe.

lected the xenon-enhanced DE CT data of 25 patients for FAN modeling. Since the current version of the FAN model does not reflect interlobar collateral ventilation, we excluded three cases with incomplete fissure boundaries. A total of twenty-two men with COPD (mean age, 67 years \pm 7 [standard deviation]) were evaluated. The severity of COPD ranged from stage II to stage IV according to the Global Initiative for Chronic Obstructive Lung Disease classification (23). Table 1 shows a summary of participant demographics and their PFT results.

\section{Ventilation Distribution}

Three-dimensional ventilation distribution computed in the FAN flow model is demonstrated in Figure 2, $C$. To better visualize the intuitive comparison, Figure 3 shows midtransverse and midcoronal planes of structural CT scans and the corresponding xenon ventilation maps assessed with xenon-enhanced DE CT and the FAN model in five study participants. Defect size and locations associated with ventilation distribution heterogeneity and the severity of disease varied among patients. Central areas of lungs were relatively well ventilated compared with peripheral areas in most patients except the one shown in Figure 3, $C$. We found a close visual similarity in ventilation distribution between xenon-enhanced DE CT and FAN. The structural similarity index of FAN-modeled ventilation on the cross-sectional planes appears similar to the xenon-enhanced DE CT data. The structural similarity index ranges in the midtransverse and midcoronal planes were $0.79-0.87$ and $0.79-0.90$, respectively.

Regional ventilation estimated with xenon-enhanced DE CT imaging data and the FAN model were compared with their percentage lobar ventilation (Appendix E1 [online]) and the CV of ventilation distribution, as demonstrated in Figure 4. The percentage lobar ventilation and the linear regression plot in Figure 4, $A$, show strong positive correlation between the xenon-enhanced DE CT and FAN estimations. The Lin concordance correlation coefficient $\left(\rho_{\mathrm{c}}=0.7, P<.001\right)$ and the Pearson correlation coefficient $(r=0.7, P<.001)$ were obtained. In addition to ventilation, the heterogeneity of lobar ventilation distribution was compared with the Bland-Altman plot (Fig 4, B). This showed the majority (94.5\%) of the lobar CV differences lied within $95 \%$ limits of agreement (-0.45 and 0.29). Mean bias and standard deviation was $-0.08 \pm 0.37$. To compare intralobar ventilation distribution, Table 2 summarizes the volume of lobar ventilation defects (less than the fifth percentile) and shows a significant association between xenon-enhanced DE CT and the FAN model.

Figure 5 shows the linear regression of the percentage emphysema and normal tissue in lungs (Appendix E1 [online]) to the relative percentage lobar ventilation in all study participants. The lobar emphysema ratio revealed weak negative correlations with 

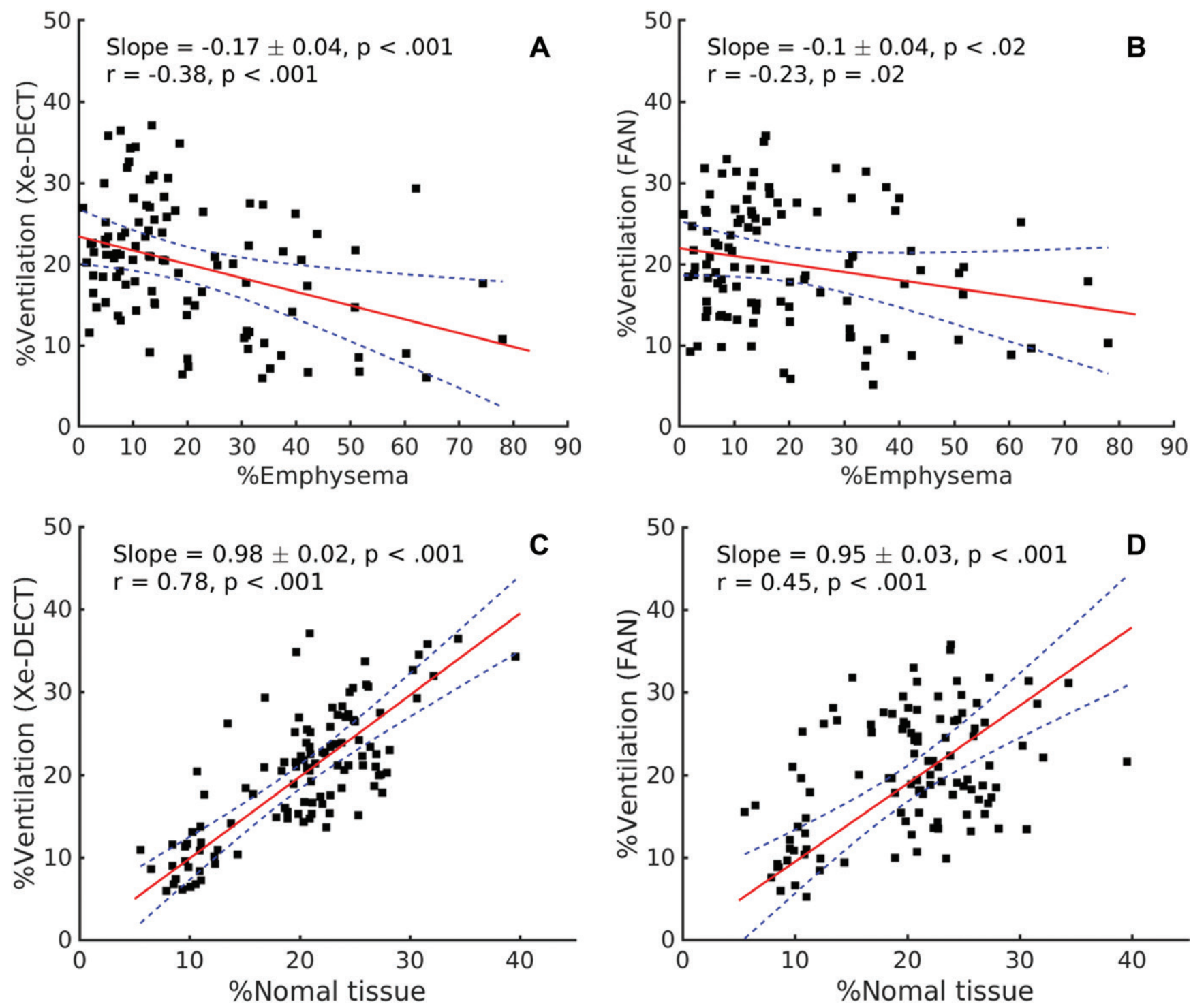

Figure 5: Comparison of relative percentage lobar emphysema and percentage lobar ventilation assessed with, $A$, xenon-enhanced dual-energy CT (Xe-DECT) and, $B$, the full-scale airway network (FAN). Comparison of percentage lobar normal tissue volume and percentage lobar ventilation assessed with, $C$, xenon-enhanced DE CT and, $D$, the FAN model.

the percentage lobar ventilation obtained with xenon-enhanced DE CT $(r=-0.38, P<.001)$ and the FAN model $(r=-0.23$, $P=.02)$. In the meantime, the lobar normal tissue ratio showed positive and stronger correlations with the percentage lobar ventilation assessed with xenon-enhanced DE CT $(r=0.78, P<$ $.001)$ and the FAN model $(r=0.45, P<.001)$ than the emphysema ratio.

In addition to the lobar analysis, we conducted analysis of the whole lung. The influence of emphysema on lung ventilation heterogeneity is shown in Figure 6, $A$ and $B$. The emphysema ratio was strongly positively correlated with the CVs from xenon-enhanced DE CT $(r=0.81, P<.001)$ and the FAN model $(r=0.59, P=.004)$. The negative correlation between heterogeneity in whole-lung ventilation and the participants' PFT results is shown in Figure 6, $C-F$. Pearson correlation coefficients between the ventilation $\mathrm{CV}$ assessed with xenon-enhanced DE CT and $\mathrm{FEV}_{1} \%(r=-0.7, P<.001)$ and $\mathrm{FEV}_{1} / \mathrm{FVC}$ ratio $(r=-0.76$,
$P<.001)$ were obtained. Pearson correlation coefficients between $\mathrm{CV}$ computed with the FAN model and $\mathrm{FEV}_{1} \%(r=-0.75, P<$ $.001)$ and between $\mathrm{CV}$ and $\mathrm{FEV}_{1} / \mathrm{FVC}$ ratio $(r=-0.67, P<.001)$ were obtained.

\section{Discussion}

The full-scale airway network (FAN) flow model uses static structural information acquired from thoracic CT scans to assess dynamic lung ventilation (13). We have previously demonstrated a strong correlation between the FAN model and functional imaging (hyperpolarized xenon $129 \mathrm{MRI}$ and ventilation SPECT) in patients with chronic obstructive pulmonary disease (14), but its correlation with functional CT data has not previously been demonstrated. In this study, we have compared FANmodeled ventilation with xenon-enhanced dual-energy CT ventilation imaging data. This approach removes the inaccuracies of the need for co-registration and ensures that the modeled data 

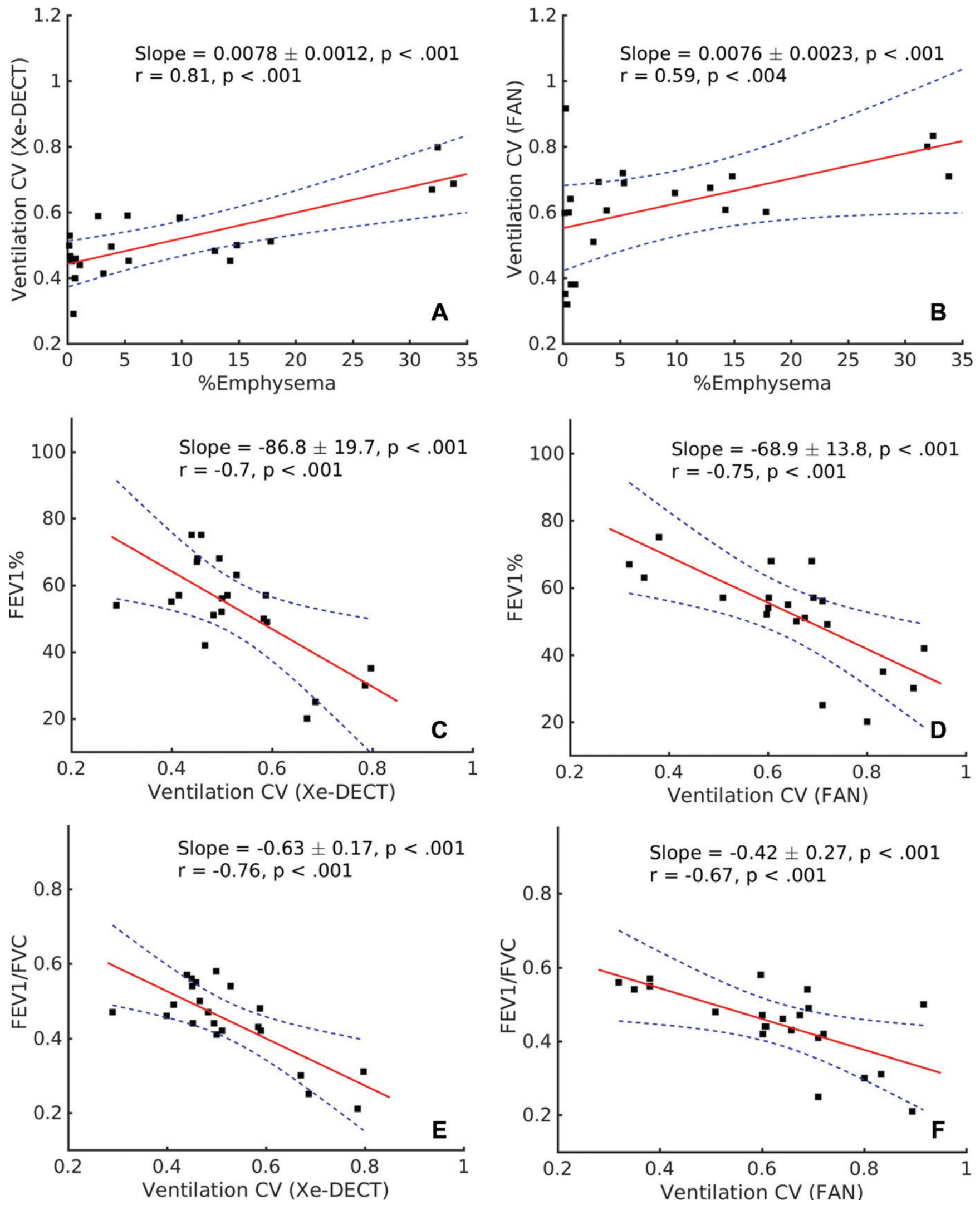

Figure 6: Lung analysis shows the relationship between emphysema $(<-950 \mathrm{HU})$ and ventilation coefficient of variation (CV) assessed with, $A$, xenon-enhanced dualenergy CT (Xe-DECT) and, B, the full-scale airway network (FAN) model. Relationship between ventilation CV and pulmonary function percentage predicted forced expiratory volume in 1 second $(F E V, \%)$ and ratio of forced expiratory volume in 1 second to forced vital capacity $(F E V, / F V C)$ assessed with $C$, xenon-enhanced dual-energy CT and $\mathrm{FEV} \%$, D, FAN and $\mathrm{FEV} \%$, E, xenon-enhanced dual-energy $\mathrm{CT}$ and $\mathrm{FEV} \% / \mathrm{FVC} \%$, and $\mathrm{F}$, FAN and $\mathrm{FEV}{ }_{1} / \mathrm{FVC}$. 
can be compared with ventilation data acquired with the same machine at the same time.

The results showed a significant correlation between relative lobar percentage ventilation assessed with FAN and xenon-enhanced DE CT data $\left(\rho_{c}=0.7, P<.001 ; r=0.7, P<.001\right)$. The relative lobar percentage ventilation was negatively correlated with percentage emphysema but positively correlated with percentage normal tissue volume. Additionally, spatial heterogeneities quantified with CV enabled confirmation of good agreement with lobar ventilation distribution between xenon-enhanced DE CT imaging and FAN flow modeling. Lung ventilation CVs of FAN revealed negative correlations with the spirometry results $\left(\mathrm{CV}_{\mathrm{FAN}}\right.$ vs $\mathrm{FEV}_{1} \%: r=-0.75, P<.001 ; \mathrm{CV}_{\text {FAN }}$ vs $\mathrm{FEV}_{1} / \mathrm{FVC} \%: r=-0.67$, $P<.001)$. This lumped approach to assess lung function with spirometry smooths regional lung function, losing regional variation. The FAN model has the potential to elucidate regional ventilation characteristics in future studies.

In a recent study presenting a technique to assess lobar ventilation with HPX MRI, Doganay et al compared lobar ventilation estimated from HPX MRI data in a COPD cohort with that obtained from V-SPECT images (24). They found a strong correlation $(r=0.64, P<.001)$ between HPX MRI and VSPECT imaging assessment. In an alternative approach to measure regional ventilation per lobe, Tahir et al calculated CT lobar volume expansion and compared it with hyperpolarized helium 3 MRI data (25). Their CT- and MRI-based lobar ventilation assessments showed strong correlation $(r=0.65, P<.001)$.

In a previous study, the percentage lobar area with low attenuation $(<-950 \mathrm{HU})$ was negatively correlated with HPX MRI ventilation volume $(r=-0.32, P=.00061)$ (4). We found similarly weakly negative correlations between the percentage of emphysema and the percentage lobar ventilation assessed with xenon-enhanced DE CT and the FAN flow model. In contradistinction to this, correlations of the percentage normal tissue and percentage lobar ventilations were positive and stronger. Our results suggest that the influence of healthy tissue on lobar ventilation is more significant than the effect of emphysema on reducing ventilation. Thus, measuring the amount of normal tissue may be more useful in evaluating regional lung function than measuring the degree of emphysema or ventilation defects, at least in patients with moderate to severe COPD according to Global Initiative for Chronic Obstructive Lung Disease criteria.

A study with HPX MRI in healthy lungs and lungs affected by COPD identified a strongly positive correlation $(r=0.82, P$ $<$.0001) between the ventilation defect score and CV (26). In a separate study with hyperpolarized helium 3 MRI, Svenningsen et al reported a significant correlation between ventilation defects identified with MRI and the lung clearance index, identifying significant ventilation heterogeneity $(r=0.86, P<.0001)(27)$. We have also shown good correlation between the percentage of lung emphysema and CV for both xenon-enhanced DE CT and the FAN model data (xenon-enhanced DE CT: $r=0.81, P<$ .001 ; FAN: $r=0.59, P=.004)$. Virgincar et al showed a negative correlation of CV and $\mathrm{FEV}_{1}(r=-0.66, P=.0059)$ in HPX MRI analysis (26). We have demonstrated a comparable relationship between ventilation $\mathrm{CV}$ and pulmonary function parameters for both xenon-enhanced DE CT and the FAN model data.
Our study had several limitations. First, the sample size for modeling was small, and the study population was unintentionally biased toward male sex. Second, the FAN modeling process requires optimization to be available for clinical use. Third, lung segments were not analyzed from their bronchial branching pattern to further refine the regionality of these ventilation data; we will do this in future iterations of this method. Finally, we assumed that the VNC xenon-enhanced DE CT images were similar to native nonenhanced CT scans of the chest $(7,9)$.

In conclusion, we have successfully derived pulmonary ventilation using the structural information extracted from xenon-enhanced dual-energy (DE) CT with the full-scale airway network (FAN) flow model. The FAN-modeled ventilation showed close correlation with the xenon-enhanced DE CT imaging data, with lung densitometry and pulmonary function tests. The FAN flow model has the potential to investigate the regional distribution of small-airway disease and further quantify the severity or grade of obstructive lung disease. These new metrics derived from routine nonenhanced CT could help to select and monitor patients in research trials or a clinical setting.

Author contributions: Guarantors of integrity of entire study, M.K., O.D., F.V.G.; study concepts/study design or data acquisition or data analysis/interpretation, all authors; manuscript drafting or manuscript revision for important intellectual content, all authors; approval of final version of submitted manuscript, all authors; agrees to ensure any questions related to the work are appropriately resolved, all authors; literature research, M.K., O.D.; clinical studies, M.K., H.J.H., J.B.S.; statistical analysis, M.K., O.D.; and manuscript editing, all authors

Disclosures of Conflicts of Interest: M.K. disclosed no relevant relationships. O.D. disclosed no relevant relationships. H.J.H. disclosed no relevant relationships. J.B.S. disclosed no relevant relationships. F.G. disclosed no relevant relationships.

\section{References}

1. Mathers CD, Loncar D. Projections of global mortality and burden of disease from 2002 to 2030. PLoS Med 2006;3(11):e442.

2. Mathew L, Kirby M, Etemad-Rezai R, Wheatley A, McCormack DG, Parraga G. Hyperpolarized ${ }^{3} \mathrm{He}$ magnetic resonance imaging: preliminary evaluation of phenotyping potential in chronic obstructive pulmonary disease. Eur J Radiol 2011;79(1):140-146.

3. Kirby M, Svenningsen S, Owrangi A, et al. Hyperpolarized 3He and 129Xe MR imaging in healthy volunteers and patients with chronic obstructive pulmonary disease. Radiology 2012;265(2):600-610.

4. Matin TN, Rahman N, Nickol AH, et al. Chronic Obstructive Pulmonary Disease: Lobar Analysis with Hyperpolarized 129Xe MR Imaging. Radiology 2017;282(3): 857-868.

5. Marshall H, Deppe MH, Parra-Robles J, et al. Direct visualisation of collateral ventilation in COPD with hyperpolarised gas MRI. Thorax 2012;67(7):613-617.

6. Bajc M, Chen Y, Wang J, et al. Identifying the heterogeneity of COPD by V/P SPECT: a new tool for improving the diagnosis of parenchymal defects and grading the severity of small airways disease. Int J Chron Obstruct Pulmon Dis 2017;12. 1579-1587.

7. Lee SM, Seo JB, Hwang HJ, et al. Assessment of regional emphysema, air-trapping and Xenon-ventilation using dual-energy computed tomography in chronic obstructive pulmonary disease patients. Eur Radiol 2017;27(7):2818-2827.

8. Park E-A, Goo JM, Park SJ, et al. Chronic obstructive pulmonary disease: quantitative and visual ventilation pattern analysis at xenon ventilation CT performed by using a dual-energy technique. Radiology 2010;256(3):985-997.

9. Hwang HJ, Seo JB, Lee SM, et al. Assessment of regional xenon ventilation, perfusion, and ventilation-perfusion mismatch using dual-energy computed tomography in chronic obstructive pulmonary disease patients. Invest Radiol 2016;51(5):306315 .

10. Kong X, Sheng HX, Lu GM, et al. Xenon-enhanced dual-energy CT lung ventilation imaging: techniques and clinical applications. AJR Am J Roentgenol 2014;202(2):309-317

11. Honda N, Osada H, Watanabe W, et al. Imaging of ventilation with dual-energy CT during breath hold after single vital-capacity inspiration of stable xenon. Radiology 2012;262(1):262-268

12. Chae EJ, Seo JB, Lee J, et al. Xenon ventilation imaging using dual-energy computed tomography in asthmatics: initial experience. Invest Radiol 2010;45(6):354-361.

13. Kim M, Bordas R, Vos W, et al. Dynamic flow characteristics in normal and asthmatic lungs. Int J Numer Methods Biomed Eng 2015;31(12):e02730. 
14. Kim M, Doganay O, Matin TN, Povey T, Gleeson FV. CT-based Airway Flow Model to Assess Ventilation in Chronic Obstructive Pulmonary Disease: A Pilot Study. Radiology 2019;293(3):666-673.

15. Schiebler ML, Parraga G. Visualization of the small airways: What it is and why it matters. Radiology 2019;293(3):674-675.

16. Doel T. The pulmonary toolkit. https://github.com/tomdoel/pulmonarytoolkit. Accessed December 10, 2018.

17. Bordas R, Lefevre C, Veeckmans B, et al. Development and analysis of patient-based complete conducting airways models. PLoS One 2015;10(12):e0144105.

18. Geuzaine C, Remacle JF. Gmsh: A 3-D finite element mesh generator with built-in preand post-processing facilities. Int J Numer Methods Eng 2009;79(11):1309-1331.

19. Wang Z, Bovik AC, Sheikh HR, Simoncelli EP. Image quality assessment: from error visibility to structural similarity. IEEE Trans Image Process 2004;13(4):600-612.

20. Kirby M, Svenningsen S, Kanhere N, et al. Pulmonary ventilation visualized using hyperpolarized helium-3 and xenon-129 magnetic resonance imaging: differences in COPD and relationship to emphysema. J Appl Physiol (1985) 2013;114(6):707-715.

21. Cressoni M, Gallazzi E, Chiurazzi C, et al. Limits of normality of quantitative thoracic CT analysis. Crit Care 2013;17(3):R93.
22. Ohkubo H, Kanemitsu Y, Uemura T, et al. Normal lung quantification in usual interstitial pneumonia pattern: The impact of threshold-based volumetric CT analysis for the staging of idiopathic pulmonary fibrosis. PLoS One 2016;11(3):e0152505 [Published correction appears in PLoS One 2016;11(8):e0160231.].

23. Vestbo J, Hurd SS, Agustí AG, et al. Global strategy for the diagnosis, management, and prevention of chronic obstructive pulmonary disease: GOLD executive summary. Am J Respir Crit Care Med 2013;187(4):347-365.

24. Doganay O, Matin T, Chen M, et al. Time-series hyperpolarized xenon-129 MRI of lobar lung ventilation of COPD in comparison to V/Q-SPECT/CT and CT. Eur Radiol 2019;29(8):4058-4067.

25. Tahir BA, Van Holsbeke C, Ireland RH, et al. Comparison of CT-based Lobar Ventilation with $3 \mathrm{He}$ MR Imaging Ventilation Measurements. Radiology 2016;278(2): 585-592.

26. Virgincar RS, Cleveland ZI, Kaushik SS, et al. Quantitative analysis of hyperpolarized 129 Xe ventilation imaging in healthy volunteers and subjects with chronic obstructive pulmonary disease. NMR Biomed 2013;26(4):424-435.

27. Svenningsen S, Nair P, Guo F, McCormack DG, Parraga G. Is ventilation heterogeneity related to asthma control? Eur Respir J 2016;48(2):370-379. 